\title{
The use of a lesion score as an indicator for agonistic behaviour in pigs
}

Andreas Stukenborg', Imke Traulsen', Eckhard Stamer ${ }^{2}$, Birger Puppe ${ }^{3}$ and Joachim Krieter $^{1}$

${ }^{1}$ Institute of Animal Breeding and Husbandry, Christian-Albrechts-Universität zu Kiel, Kiel, Germany, ${ }^{2}$ TiDa Tier und Daten $\mathrm{GmbH}$, Westensee/Brux, Germany, ${ }^{3}$ Research Unit Behavioural Physiology, Leibniz Institute for Farm Animal Biology (FBN), Dummerstorf, Germany

\begin{abstract}
The aim of the present study was to analyse whether a lesion score approach could be used as an indicator for agonistic behaviour in pigs. Behavioural patterns were observed directly after weaning (PIG-28, $\mathrm{n}=827$ ), 40 days later (growing pigs, PIG-68, $\mathrm{n}=292$ ) and immediately after mixing gilts (GILTS, $n=344$ ), over a $48 \mathrm{~h}$ period. For the front, middle and rear part of the body the extent of skin injuries was assessed with a lesion score (LS). The LS ranked from 0 (no wounds) to 4 (many, deep wounds) and was determined at the beginning (LSstart) and at the end (LSend) of the observation period. In all groups, the front body third was mostly stressed by fighting interactions. The highest correlations were found between the front LS and the overall fight time, with $r=0.50$ (PIG-28), $r=0.52$ (PIG-68) and $r=0.48$ (GILTS). The LS for the front area did not increase for $26 \%$ (PIG-28), 39\% (PIG-68) and $40 \%$ (GILTS) of the animals. According to this, the applied LS approach only reflected the agonistic behaviour of pigs to a certain extent.
\end{abstract}

Keywords: pig, agonistic behaviour, lesion score

\section{Introduction}

Agonistic behaviour in pigs especially occurs when unacquainted pigs are mixed with groups of acquainted ones. Fighting often results in the accumulation of skin lesions which can have detrimental effects on the welfare and longevity of the animals (Spoolder et al. 2000, Løvendahl et al. 2005). In recent studies, a skin lesion score (lesion score, LS) was applied as a proxy indicator for the aggressive potential of a pig (Turner et al. 2006). With regard to the results in Turner et al. $(2008,2009)$ the LS is a heritable trait and genetically correlated with aggressive behaviour. They concluded that a selection based on breeding values for a reduced LS is expected to reduce reciprocal aggression (Turner et al. 2008). However, most of the previous applied lesion scores have implemented detailed counting or assessment of each scratch (e.g. Erhard et al. 1997, Weng et al. 1998, Spoolder et al. 2000, Turner et al. 2006). Thus, they seem to be infeasible to measure the LS under common breeding farm conditions. The aim of this study was to apply an easy and rapid lesion score application under commercial sow farm conditions. The behaviour and lesion score recordings were made in three age groups. Finally, the study examined whether the LS could be an indicator 
for agonistic behaviour in pigs. Therefore, correlations were estimated between the LS and agonistic behavioural traits.

\section{Materials and methods}

\section{Animals and housing}

The data collection was carried out on a closed herd sow farm of the German breeding company »Hülsenberger Zuchtschweine« during October 2007 to April 2009. The herd composed of pure-bred Large-White (LW) sows and also crosses between Large-White and German Landrace $(\mathrm{LW} \times \mathrm{DL})$. For the present study, female pigs from pure bred litters and crosses from $\mathrm{LW} \times \mathrm{DL}$ were considered. Behavioural observations from weaned (PIG-28) piglets and growing pigs (PIG-68) were made in the rearing pens. Every Wednesday piglets from one sow group with 36 litters on average were weaned (one-week rhythm) and moved to the rearing house where they were sorted by gender and body size. 29 piglets on average were housed in one rearing pen. The pens had dimensions of $5.10 \mathrm{~m} \times 2.10 \mathrm{~m}$. They had a fully slatted floor and were separated by $60 \mathrm{~cm}$ high, closed partitions on the ground and two rods across. During the rearing period water was non-stop accessible on nipple drinkers. Feed was provided ad libitum with a wet feeder. After the rearing period the pigs were taken to a fattening unit where the own performance test was implemented. The pigs were fed with a liquid feeding system three times a day. The third observation was carried out when the gilts (GILTS) were brought back to the sow farm directly after the first mixing in the pregnancy area. An average of 48 gilts was housed as a separate group in the gilt pen with dimensions of ca. $15.5 \mathrm{~m} \times 6.5 \mathrm{~m}$. The pen was divided into a feeding (ca. $\left.43 \mathrm{~m}^{2}\right)$ and a lying area $\left(\right.$ ca. $58 \mathrm{~m}^{2}$ ). The feeding area included the electronic sow feeding station and had a fully slatted floor. The lying area comprised a corridor with a fully slatted floor and six resting areas (each ca. $5.4 \mathrm{~m}^{2}$ ) with a solid floor. Water was accessible ad libitum on one nipple drinker and two water troughs.

\section{Behavioural observation}

Four rearing pens with female breeding piglets were filmed directly after weaning (PIG-28) using the HeiTel Player software program (HeiTel Digital Video GmbH, Kiel, Germany). The behavioural observations were made for 10 focus piglets per pen, which were randomly chosen and individually marked on their backs. The observation area excluded the area around the wet feeder and measured $3.20 \mathrm{~m} \times 2.10 \mathrm{~m}$. A second observation took place 40 days after weaning (growing pigs=PIG-68). A new group was created in equal shares from four pens which were previously housed at weaning. The same 40 focus pigs which had already been observed at weaning were re-marked, re-mixed and re-allocated to the four rearing pens. Thus, one out of four from the new group and also two or three from the marked pigs were already known to each other. The third observation was carried out in the gestation house (GILTS). Before the gilts were moved into the pen, each animal was individually marked. The recording period started immediately after housing the gilts. The behaviour of all animals inside the whole pen area was observed. The agonistic behaviour of 
each marked pig was evaluated with the aid of the videotapes. A single agonistic interaction was defined as a fight or a displacement with physical contact initiated by one individual and featuring aggressive behavioural elements, followed by any form of submissive behaviour performed by the opponent (Langbein \& Puppe 2004). The aggressor/receiver, the winner/ loser of a fight and also the times (start time and finish time) were noted for each fight in which a marked pig was involved (for a more detailed description see Stukenborg et al. 2011). Based on the beginning and outcome of the fight, the values of ten agonistic behavioural traits were calculated for each observed pig (Table 1).

Table 1

Definitions of the agonistic behaviour traits

\begin{tabular}{|c|c|c|}
\hline Behavioural trait & Unit & Definition \\
\hline Fights & no. of & $\begin{array}{l}\text { Number of all agonistic interactions per pig } \\
\text { in which the pig was involved }\end{array}$ \\
\hline Time Fights & seconds, s & $\begin{array}{l}\text { The total time a marked pig was involved in agonistic } \\
\text { interactions during the } 48 \mathrm{~h} \text { observation period }\end{array}$ \\
\hline Aggressive Fights & no. of & $\begin{array}{l}\text { The number of fights per pig in which the pig initiated } \\
\text { the agonistic interaction }\end{array}$ \\
\hline Time Aggressive Fights & seconds, s & $\begin{array}{l}\text { The total time a marked pig was involved in self-initiated } \\
\text { agonistic interactions during the } 48 \mathrm{~h} \text { observation period }\end{array}$ \\
\hline Aggressions Received & no. of & $\begin{array}{l}\text { The number of fights per pig in which the pig was subjected } \\
\text { to (not the initiator of) the agonistic interaction }\end{array}$ \\
\hline Time Aggressions Received & seconds, s & $\begin{array}{l}\text { The total time a pig was subjected to agonistic interactions } \\
\text { during the } 48 \mathrm{~h} \text { observation period }\end{array}$ \\
\hline Fights Won & no. of & $\begin{array}{l}\text { The number of fights per pig in which the pig won the } \\
\text { agonistic interaction }\end{array}$ \\
\hline Time Fights Won & seconds, s & $\begin{array}{l}\text { The total time a marked pig was involved in won agonistic } \\
\text { interactions during the } 48 \mathrm{~h} \text { observation period }\end{array}$ \\
\hline Fights Lost & no. of & $\begin{array}{l}\text { The number of fights per pig in which the pig lost the } \\
\text { agonistic interaction }\end{array}$ \\
\hline Time Fights Lost & seconds, s & $\begin{array}{l}\text { The total time a pig was involved in lost agonistic interactions } \\
\text { during the } 48 \mathrm{~h} \text { observation period }\end{array}$ \\
\hline
\end{tabular}

\section{Lesion score}

A skin lesion score (LS) was assigned by one person to all pigs marked (LSstart) immediately before starting the $48 \mathrm{~h}$ observation period. After $48 \mathrm{~h}$ (PIG-68) or one week later (PIG-28 and GILTS), the LS was recorded again (LSend) for the observed pigs. The time differences between the first and second recordings ( $48 \mathrm{~h}$ vs. one week) were necessary in order not to disturb the workflow on the farm. Three body regions were evaluated independently of each other: front (head, neck, shoulders and front legs), middle (flanks and back) and rear (rump, hind legs and tail) (modified according to Turner et al. 2006). A detailed counting of each scratch on the pigs' bodies was not carried out. Although such a procedure would probably have given an exact indication of the new wounds, the counting of the lesions appeared to be too time-consuming for use under commercial farm conditions. The applied LS ranked from 0 to 4 as shown in Table 2. 
Finally, because of the missing of culled or diseased pigs for LSend recording and due to problems with the technical supply, both lesion scores as well as behavioural observations were ascertained for 827 (PIG-28), 292 (PIG-68) and 344 (GILTS) animals.

Table 2

Description of the lesion score ranking

\begin{tabular}{ll}
\hline Code & Description \\
\hline 0 & No skin injuries (no lesions on the whole body area) \\
1 & Minor skin injuries (sporadically occurring) lesions on the body area) \\
2 & Middle skin injuries (several lesions allocated over the whole body area; \\
3 & no widespread accumulation of injuries) \\
4 & $\begin{array}{l}\text { Strong skin injuries (several lesions allocated over the whole body area } \\
\text { with accumulations of injuries at different areas of the skin }\end{array}$ \\
\hline
\end{tabular}

\section{Statistical analysis}

In order to reach approximately normal distributions, the behaviour data were transformed with a square root (for PIG-28) or log transformation Y=loge (1+observation) (for PIG-68 and Gilts). All further analyses were based on the transformed behaviour data.

An analysis of fixed effects was performed with the MIXED procedure in SAS 9.1 (SAS Institute Inc., Cary, NC, USA). For model fitting, the information criteria of Hurvich and Tsai (1989) (Akaike information criterion, AIC) and Schwarz (1978) (Bayesian information criterion, $\mathrm{BIC})$ were considered. These values take into account the number of estimated parameters and favour less complex model variants. The model with the smallest AIC and BIC values was chosen without considering the underlying significance of fixed effects. For the behavioural traits, the final model for PIG-28 and PIG-68 included the fixed effect of the observation month and the random pen and litter effect. For GILTS behaviour, the fixed effect of the observation date was considered. For LSend, the same models were used for behavioural traits expanded by the fixed effect of LSstart. Pearson correlation coefficients were calculated between the residual of the behavioural traits and LSend (Proc CORR; SAS 9.1, [SAS Institute Inc., Cary, NC, USA]).

\section{Results}

\section{Behavioural performance}

Most fighting interactions were observed for weaned piglets (Table 3). GILTS had the lowest median number of fights. However, the total fight time was considerably longer in comparison to growing pigs. Great differences in fighting behaviour were also seen within age groups. In all age classes at least one pig was not involved in agonistic interaction for the first $48 \mathrm{~h}$. In contrast, the most active animals had an overall fight time approximately 220 (PIG-28), 113 (PIG-68) and 108 (GILTS) min, respectively. A more detailed description of agonistic behaviour in weaned piglets and growing pigs is given in Stukenborg et al. (2011). 
Table 3

Median of the untransformed behavioural traits per animal for each age group

\begin{tabular}{lrlrcrl}
\hline & \multicolumn{2}{c}{ PIG-28 } & \multicolumn{2}{c}{ PIG-68 } & \multicolumn{2}{c}{ GILTS } \\
& Median & Min/Max & Median & Min/Max & Median & Min/Max \\
\hline Fights") & 39 & $0 / 188$ & 8 & $0 / 86$ & 6 & $0 / 33$ \\
Time Fights & 2460 & $0 / 13171$ & 99 & $0 / 6751$ & 280 & $0 / 6481$ \\
Aggressive Fights & 13 & $0 / 140$ & 2 & $0 / 51$ & 2 & $0 / 17$ \\
Time Aggressive Fights & 781 & $0 / 7083$ & 18 & $0 / 2500$ & 86 & $0 / 2992$ \\
Aggressions Received & 14 & $0 / 57$ & 4 & $0 / 25$ & 3 & $0 / 16$ \\
Time Aggressions Received & 712 & $0 / 7076$ & 47 & $0 / 3605$ & 102 & $0 / 3640$ \\
Fights Won & 5 & $0 / 78$ & 0 & $0 / 41$ & 1 & $0 / 16$ \\
Time Fights Won & 214 & $0 / 2658$ & 0 & $0 / 1344$ & 33 & $0 / 2560$ \\
Fights Lost & 5 & $0 / 63$ & 2 & $0 / 15$ & 2 & $0 / 13$ \\
Time Fights Lost & 246 & $0 / 3039$ & 22 & $0 / 1700$ & 77 & $0 / 2190$ \\
\hline
\end{tabular}

1)Descriptions for the behaviour traits are given in Table 1.
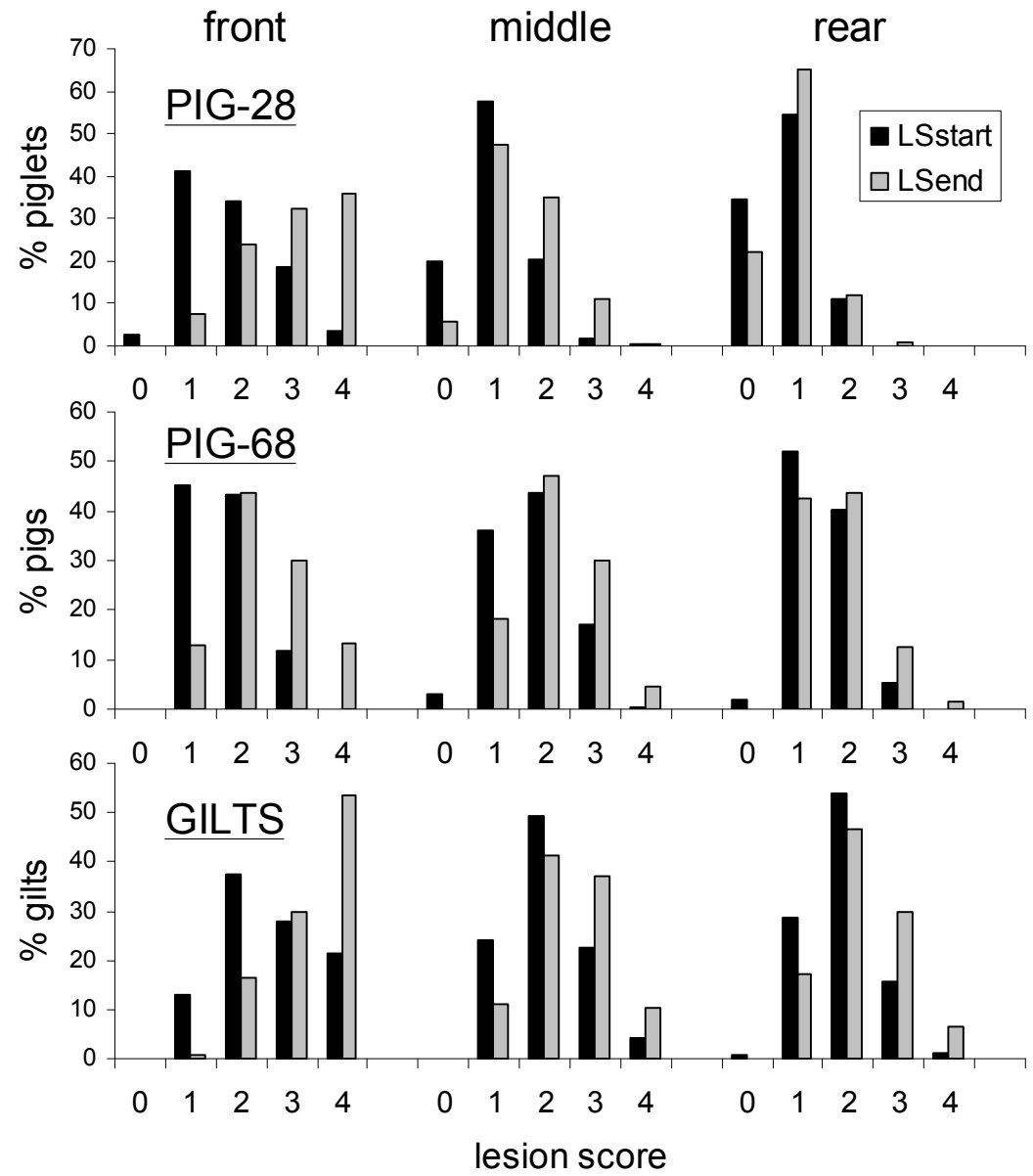

Figure 1

Distribution of the first and second LS for the body regions of each age group 


\section{Lesion score differences}

Figure 1 shows the distribution of the first and second LS for the body regions of each age group. In general an LSstart equal to zero was rarely observed for growing pigs and gilts. The LS for the front body region increased (LSstart<LSend) for 74\% (PIG-28), $61 \%$ (PIG-68), and $60 \%$ (GILTS) of the animals, respectively (results not presented). However, almost $50 \%$ of gilts had an LSstart greater than 2. Mostly small variations were observed between LSstart and LSend for the middle body area. The majority of the weaned piglets had a middle LSstart of 1. In comparison, PIG-68 and GILTS had higher starting values overall. About $50 \%$ (PIG-28/PIG68) and $56 \%$ (GILTS) of the pigs had no positive change in the middle LS. For the rear body area, most LS differences were observed for gilts. In contrast to the younger age groups, a considerable number of gilts had lesion scores of 3 or 4 . However, an increased LS was only shown for nearly $29 \%$ (PIG-28), $33 \%$ (PIG-68) and $42 \%$ (GILTS) of the pigs, respectively.

\section{Correlation between LS and behavioural parameters}

The highest correlation between agonistic behavioural parameters and the LSend in all age groups were shown for the front body area (Table 4). The maximum correlation coefficients were found with the overall fight time, $r=0.50$ (PIG-28) $(P<0.05), r=0.52$ (PIG-68) $(P<0.05)$ and $\mathrm{r}=0.48$ (GILTS) $(P<0.05)$. Intermediate correlations were calculated for the middle body region. The correlation coefficients ranked between $r=-0.11$ up to 0.26 (PIG-28), $r=0.11$ up to 0.29 (PIG-68) and $r=0.13$ up to 0.30 (GILTS). No significant correlation for the rear area was found for weaned piglets. The highest significant relationships between the rear LS and behavioural parameters were calculated for GILTS $(r=0.33$ for Time Fights and Time Fights Won $(P<0.05))$.

Table 4

Pearson correlation coefficients between agonistic behavioural traits and the lesion score of three different body areas observed in three different age groups (weaned piglets=PIG-28, growing pig=PIG-68, gilts=GILTS)

\begin{tabular}{lccccccccc}
\hline & \multicolumn{3}{c}{ PIG-28 } & & \multicolumn{3}{c}{ PIG-68 } & & $\begin{array}{c}\text { GILTS } \\
\text { middle }\end{array}$ \\
& front & middle & rear & front & middle & rear & front & middear \\
\hline Fights & $-0.27^{*}$ & $-0.14^{*}$ & -0.00 & $-0.49^{*}$ & $-0.25^{*}$ & $-0.17^{*}$ & $-0.37^{*}$ & $-0.21^{*}$ & $-0.25^{*}$ \\
Time Fights & $-0.50^{*}$ & $-0.26^{*}$ & -0.01 & $-0.52^{*}$ & $-0.27^{*}$ & $-0.21^{*}$ & $-0.48^{*}$ & $-0.30^{*}$ & $-0.33^{*}$ \\
Aggressive Fights & $-0.16^{*}$ & $-0.09^{*}$ & -0.01 & $-0.42^{*}$ & $-0.28^{*}$ & $-0.11^{*}$ & $-0.33^{*}$ & $-0.21^{*}$ & $-0.27^{*}$ \\
Time Aggressive Fights & $-0.34^{*}$ & $-0.19^{*}$ & -0.01 & $-0.45^{*}$ & $-0.29^{*}$ & $-0.13^{*}$ & $-0.37^{*}$ & $-0.23^{*}$ & $-0.30^{*}$ \\
Aggressions Received & $-0.15^{*}$ & $-0.07^{*}$ & -0.00 & $-0.41^{*}$ & $-0.17^{*}$ & $-0.15^{*}$ & $-0.29^{*}$ & $-0.15^{*}$ & $-0.15^{*}$ \\
Time Aggressions Received & $-0.41^{*}$ & $-0.20^{*}$ & -0.03 & $-0.46^{*}$ & $-0.23^{*}$ & $-0.20^{*}$ & $-0.40^{*}$ & $-0.24^{*}$ & $-0.26^{*}$ \\
Fights Won & $-0.25^{*}$ & $-0.17^{*}$ & -0.03 & $-0.48^{*}$ & $-0.27^{*}$ & $-0.17^{*}$ & $-0.41^{*}$ & $-0.23^{*}$ & $-0.30^{*}$ \\
Time Fights Won & $-0.33^{*}$ & $-0.20^{*}$ & -0.00 & $-0.49^{*}$ & $-0.27^{*}$ & $-0.15^{*}$ & $-0.45^{*}$ & $-0.24^{*}$ & $-0.33^{*}$ \\
Fights Lost & $-0.16^{*}$ & $-0.11^{*}$ & -0.02 & $-0.13^{*}$ & $-0.11^{*}$ & $-0.11^{*}$ & $-0.15^{*}$ & $-0.13^{*}$ & $-0.04^{*}$ \\
Time Fights Lost & $-0.07^{*}$ & $-0.02^{*}$ & -0.01 & $-0.25^{*}$ & $-0.19^{*}$ & $-0.22^{*}$ & $-0.37^{*}$ & $-0.27^{*}$ & $-0.22^{*}$ \\
\hline
\end{tabular}

${ }^{*} P<0.05$

\section{Discussion}

In general, the front body area was the most stressed body region of the pigs in all age groups. Furthermore, in all age classes the front third showed the highest correlations between LS and agonistic behaviour. These results are in accordance with McGlone (1985), who indicated 
that most of the bites were targeted mainly at the ears, face and neck. A comparison between the age groups showed that the correlation coefficients of growing pigs and gilts were rather similar in contrast to the findings of weaned piglets in which no significant relationship between the rear body area and agonistic behaviour was found. Differences between the age groups might be caused through different fighting motivations and the resulting variations in the number of scratches. Previous results have indicated moderate correlation between the behaviour of growing pigs and gilts but only small relationships between the older age groups and weaned piglets (Stukenborg et al. 2012). It is therefore assumed that the behaviour of weaned piglets is especially influenced by playful manners whereas the older age groups fight in order to establish a new dominance hierarchy. Regarding this, Stukenborg et al. (2011) suggested that weaned piglets fought much more than growing pigs but their interactions were not so intensive, so a fight did not always end in injury. In all age groups, more than $50 \%$ of the pigs had no increased LS for the middle and rear body area. This fact and the consistently smaller relationships in comparison to the front LS suggest that an investigation of the rear and the middle lesion score seemed to be rather insignificant in relation to agonistic behaviour. Although the correlation between behavioural traits and the front LS were all significant and clearly showed that a higher involvement in agonistic interactions resulted in an increased $L S$, the correlation coefficients were at the maximum at the level of average. Thus, the applied LS approach did only reflect the agonistic behaviour of pigs to a certain extent. Furthermore, it must be considered that a lot of animals already had a high front LSstart. For these pigs the LS was limited concerning an indication of fighting behaviour since high fighting activity could not lead to a high difference between LSstart and LSend, Concerning gilts, even more than $20 \%$ had a front LSstart equal to 4 . For these animals further scratches in the observation period due to fighting interactions could not result in a higher $L S$. Thus, it must be considered that before keeping the gilts in the pregnancy area, the animals were taken from the fattening unit to the sow farm and were subsequently washed as a whole group. During this time the animals could already have been involved in agonistic interactions but the added wounds were assigned to the LSstart. For this reason, in further investigations the LSstart should be noted before the animals leave their familiar group and start fighting with unacquainted pigs. Previously applied LS approaches have avoided this problem by counting the number of lesions at the beginning and at the end of the observation period (Weng et al. 1998, Spoolder et al. 2000, Turner et al. 2006) or they have tried to count only fresh lesions which were received during the observation time (Erhard et al. 1997, Turner et al. 2000, Turner et al. 2009). Turner et al. (2009) found genetic correlations between the anterior $\mathrm{LS}$ and the duration in reciprocal aggression of $r=0.67$. In comparison to these findings which include laborious data collections, the present results, with correlations of approximately $\mathrm{r}=0.50 \mathrm{in}$ all age groups, are quite promising when considering the short and easy lesion score ascertainment.

In contrast to the middle and rear body region, the front body area is highly stressed by agonistic interactions in all age groups. The estimated moderate correlation coefficient between the applied front LS and the observed agonistic behavioural traits indicated that a quick and basic approach can be used for a lesion score ascertainment. However, in further studies the LSstart should be noted before the animals leave their familiar pen in order to include all newly received wounds. 


\section{References}

Erhard HW, Mendl M, Ashley DD (1997) Individual aggressiveness of pigs can be measured and used to reduce aggression after mixing. Appl Anim Behav Sci 54, 137-151

Hurvich CM, Tsai CL (1989) Regression and Time-Series Model Selection in Small Samples. Biometrika 76, 297-307

Langbein J, Puppe B (2004) Analysing dominance relationships by sociometric methods - a plea for a more standardised and precise approach in farm animals. Appl Anim Behav Sci 87, 293-315

Løvendahl P, Damgaard LH, Nielsen BL, Thodberg K, Su G, Rydhmer L (2005) Aggressive behaviour of sows at mixing and maternal behaviour are heritable and genetically correlated traits. Livest Prod Sci 93, 73-85

McGlone JJ (1985) A quantitative ethogram of aggressive and submissive behaviors in recently regrouped pigs. J Anim Sci 61, 559-565

Schwarz G (1978) Estimating the dimension of a model. Ann Statistics 6, 461-464

Spoolder HAM, Edwards SA, Corning S (2000) Aggression among finishing pigs following mixing in kennelled and unkennelled accommodation. Livest Prod Sci 63, 121-129

Stukenborg A, Traulsen I, Puppe B, Presuhn U, Krieter J (2011) Agonistic behaviour after mixing in pigs under commercial farm conditions. Appl Anim Behav Sci, 129, 28-35

Stukenborg A, Traulsen I, Stamer E, Puppe B, Presuhn U, Krieter J (2012) Heritabilities of agonistic behavioural traits in pigs and their relationships within and between different age groups. Submitted to Livest Sci

Turner SP, Ewen M, Rooke JA, Edwards SA (2000) The effect of space allowance on performance, aggression and immune competence of growing pigs housed on straw deep-litter at different group sizes. Livest Prod Sci 66, 47-55

Turner SP, Farnworth MJ, White IMS, Brotherstone S, Mendl M, Knap P, Penny P, Lawrence AB (2006) The accumulation of skin lesions and their use as a predictor of individual aggressiveness in pigs. Appl Anim Behav Sci 96, 245-259

Turner SP, Roehe R, D'Eath RB, Ison SH, Farish M, Jack MC, Lundeheim N, Rydhmer L, Lawrence AB (2009) Genetic validation of postmixing skin injuries in pigs as an indicator of aggressiveness and the relationship with injuries under more stable social conditions. J Anim Sci 87, 3076-3082

Turner SP, Roehe R, Mekkawy W, Farnworth MJ, Knap PW, Lawrence AB (2008) Bayesian analysis of genetic associations of skin lesions and behavioural traits to identify genetic components of individual aggressiveness in pigs. Behavior Genet 38, 67-75

Weng RC, Edwards SA, English PR (1998) Behaviour, social interactions and lesion scores of group-housed sows in relation to floor space allowance. Appl Anim Behav Sci 59, 307-316

Received 16 September 2011, accepted 29 December 2011.

Corresponding author:

Joachim Krieter

email: jkrieter@tierzucht.uni-kiel.de

Institute of Animal Breeding and Husbandry, Christian-Albrechts-Universität zu Kiel, Olshausenstraße 40, 24098 Kiel, Germany 